Scientific Visualization, 2020, volume 12, number 5, pages 46 - 60, DOI: 10.26583/sv.12.5.05

\title{
Computer visualization of linear surfaces with imaginary directrices
}

\author{
V.A. Korotkiy ${ }^{1}$ \\ South Ural State University (National Research University) \\ ${ }^{1}$ ORCID: 0000-0002-5266-4701, ospolina@mail.ru
}

\begin{abstract}
$\underline{\text { Abstract }}$
The article discusses a method for constructing linear surfaces based on extracting them from an elliptical linear congruence (ELC), given by four intersecting lines or collinear correspondence of flat fields. A projective-graphic algorithm for constructing a real line intersecting the imaginary ELC directrix has been proposed. The algorithm is based on the use of an image of imaginary points in the form of a special marker, which allows for the imaginary points to be used along with real points when performing constructive constructions. Extracting a surface from an ELC is reduced to a repeated application of the algorithm.

A theorem has been proved on the existence of a pencil of planes intersecting a linear algebraic surface of the order k+2 along algebraic curves of the order $\mathrm{k}$ (Theorem 1). The theorem allows constructing a skeleton of an algebraic surface of the fourth order from lines and curves of the second order. The variants of transition from linear congruence given by four straight lines to an identical congruence given by the collinear fields $\mathrm{P} \leftrightarrow \mathrm{P}^{\prime}$ have been proposed. This transition makes it possible to solve the practically important issue of designing a linear surface passing through two conical sections. The existence theorem for the collineation $\mathrm{P} \leftrightarrow \mathrm{P}^{\prime}$, drawn in the fields $\mathrm{P}, \mathrm{P}^{\prime}$ by the curves of the second order has been proved (Theorem 2).

A biaxial linear surface with constant-length generators has been considered. It has been shown that such a surface is distinguished from a linear congruence with real axes by immersing a guiding ellipse into it, the eccentricity of which is uniquely determined by the angle between the congruence axes (Theorem 3). The technological advantage of such surfaces, allowing to recommend them for use in architecture and construction, is that they are mounted from rectilinear beams or rods of the same standard size.

Examples of computer visualization of linear surfaces with imaginary and real directrices have been presented.
\end{abstract}

Keywords: linear congruence, imaginary algebraic elements, imaginary point marker, directing curve, linera quadric, collinear fields.

\section{Introduction}

A linear congruence $\operatorname{Kr}(u, v)$ is called a two-parameter set of lines intersecting predetermined intersecting straight lines $u, v$ (congruence directrices).Directrices can be real different (hyperbolic linear congruence, HLC), real coincident (parabolic linear congruence, PLC), or imaginary complex conjugate (elliptic linear congruence, ELC).

One of the methods for constructing linear surfaces is to extract them from the linear congruence by immersing a guiding curve line in the congruence body. For example, if a surface is distinguished from $\operatorname{Kr}(u, v)$ by specifying a second-order guiding curve $\tau$, we obtain a fourthorder algebraic linear surface with guides $u, v, \tau[1]$.

A constructive way of separating a surface from an HLC with the directrices $u$, $v$ consists in constructing a one-parameter set of rectilinear generators of the required surface passing through the points of the guiding curve $\tau$ and intersecting the real lines $u, v$. 
This problem is solved by the well-known methods of descriptive geometry [1]. It should be noted that a metric condition can be used instead of a guide curve. For example, a linear surface can be separated from the HLC by the requiring a constant length of the generatrix (see Section 7).

To extract a surface from a PLC, an auxiliary linear quadric $\Phi$ is introduced into consideration and a PLC is defined as a set of straight lines intersecting two infinitely close (in the limit coinciding) intersecting generators $u, v$ of the quadric $\Phi$. This allows the PLC to be identified as a family of straight lines tangent to a linear quadric at the points of one of its rectilinear generators [3].Through an arbitrarily specified point of the guiding curve $\tau$ immersed in the PLC, there is the only line $\mathrm{p}$ tangent to the quadric $\Phi$ and intersecting its generator $\mathrm{u}=\mathrm{v}$. The construction of the straight line $p$ is performed by the well-known methods of descriptive geometry [1]. The one-parameter set of straight lines $p$ forms the desired linear surface extracted from the PLC.

If it is required to extract a surface from ELC, then the problem is reduced to constructing a set of real straight lines (forming the required surface), each of which intersects a pair of imaginary complex conjugate straight lines $u \sim v$ (ELC directrices) when passing through one of the points of the guiding curve $\tau$. The methods of classical descriptive geometry are inapplicable in this case, since they involve the execution of graphic constructions with the participation of imaginary points and imaginary lines.

Graphic constructions with the participation of imaginary elements were considered both in the works of the authors of the 19th century [2] and in modern studies [3-6], but they didn't pose or solve the problem of constructing a real straight line incident to a predetermined point in space and intersecting with two predetermined imaginary conjugate crossing lines (ELC directrices). For example, in [3, p. 74], an algorithm for constructing a straight line intersecting with the imaginary ELC directrices is indicated, but the incidence requirement for this point is not taken into account.

Scientific novelty. The article proposes a projective-graphical algorithm for constructing a real line that satisfies both the condition of incidence at a predetermined point, and the condition of intersection with predetermined imaginary ELC directrices. For the constructive implementation of the proposed algorithm, computer visualization tools [7] are used for imaginary elements in conjunction with a software module [8-10], which allows drawing curves of the second order incident to any predetermined set of both real and imaginary points and tangents [11].

The relevance of the work is due to the fact that imaginary algebraic elements (imaginary points, imaginary lines, imaginary algebraic lines and surfaces) are an integral attribute of any actions with algebraic varieties. The participation of imaginary elements in geometric algorithms is just as necessary and natural as the participation of complex numbers in algebraic transformations. As a result, the problem of the exact constructive implementation of graphical algorithms containing actions with imaginary algebraic figures is urgent.

Practical significance. The proposed algorithm for extracting a linear surface from a linear congruence with imaginary directrices makes it possible to design architectural forms, floors, and shells that differ from traditional surfaces based on real axes, which expands the area of application of linear structures in architectural and construction design. In what follows, the directrices $u, v$ will be called the axes of congruence.

\section{Formulation of the problem}

A linear congruence can be specified in several different ways, in particular, by its own axes $u$, $v$ (Method 1) or by four pairwise crossing lines $a, b, c, d$ belonging to the congruence (Method 2). 
Both methods are geometrically equivalent: if 4 lines $a, b, c, d$ belonging to a congruence are given, then its axes $\mathrm{u}, \mathrm{v}$ can be found.

Let's consider an algorithm for constructing the $\mathrm{u}, \mathrm{v}$ axes of the congruence $\operatorname{Kr}(a, b, c, d)$ given in the second method. Let us introduce the linear quadric $\Phi$, which is completely defined by three of the given straight lines, for example, the straight lines $a, b, c$. The quadric $\Phi=\Phi_{1}+\Phi_{2}$ consists of the semi-quadric $\Phi_{1}$ containing the straight lines $a, b, c$, and of the conjugate semi-quadric $\Phi_{2}$ (Fig. 1).

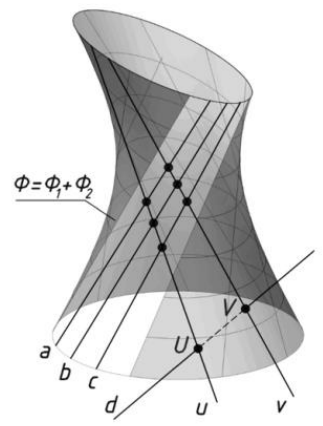

a)

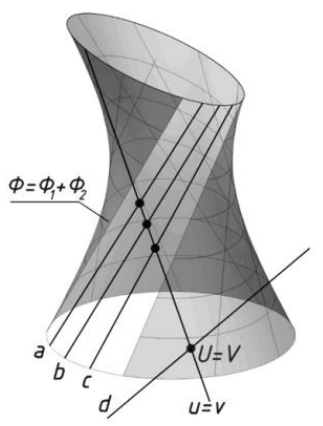

b)

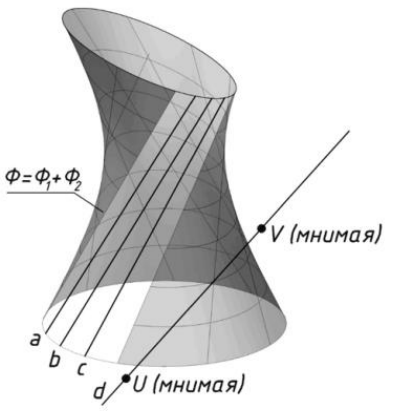

c)

Fig. 1. Constructing the axes of the congruence $\operatorname{Kr}(a, b, c, d)$ : (a) HLC; (b) PLC; (c) ELC

Let the straight line $d$ intersect the quadric $\Phi$ at the points $U, V$. Then the straight lines $u, v$ of the semi-quadric $\Phi_{2}$ passing through the points $U, V$ are the congruence axes [3].

If the straight line $d$ intersects $\Phi$ at the real points $U, V$, then the $u$ and $v$ axes are real lines, and the congruence $\operatorname{Kr}(a, b, c, d)$ is hyperbolic (Fig. 1, a). If the points $U, V$ coincide (the straight line $d$ touches the quadric $\varphi$ ), then we obtain a PLC with infinitely close (coinciding in the limit) crossed axes $u=v$ (Fig. 1, b). If the straight line $d$ intersects with $\Phi$, at real points $U, V$ and then the $\mathrm{u}$ and $\mathrm{v}$ axes are real lines, and the congruence $\mathrm{Kr}(\mathrm{a}, \mathrm{b}, \mathrm{c}, \mathrm{d})$ is hyperbolic (Fig. 1, a). If the points U, V coincide (the straight line d touches the quadric $\varphi$ ), then we obtain a PLC with infinitely close (coinciding in the limit) crossing axes $u=v$ (Fig. 1, b). If the straight line d does not intersect with the quadric $\Phi$, then the points $U, V$ and the generating $\mathrm{u}, \mathrm{v}$ of the semi-quadrics $\Phi 2$ passing through them are imaginary (Fig. 1, c).In the latter case, we obtain an elliptical linear congruence (ELC), to which the known surface extraction methods suitable for GLC and PLC are not directly applicable.

Hence the problem follows: to develop a graphical algorithm for constructing and visualizing a linear surface, separated from the ELC by immersing a guiding curve $\tau$ into it.

Only rectilinear generator $t$ of the sought surface included in this ELC passes through any given point of $T$ of the curve $\tau$. Therefore, the problem of extracting the surface from the ELC is reduced to compiling an algorithm for constructing a straight line passing through a given point $T$ and intersecting a pair of imaginary complex conjugate striaght lines $u \sim v$ (ELC directrix), and to the subsequent repeated application of this algorithm to a series of points $T_{i}$ of the guide curve $\tau$, where $i$ is the number of generators of the constructed surface required for accurate visualization.

\section{Extracting a straight line passing through a given point from an ELC}

Let a linear congruence be given by four pairwise intersecting straight lines $a, b, c, d$. It is required to extract from the congruence $\operatorname{Kr}(a, b, c, d)$ a straight line passing through the given predetermined point $T$ in the three-dimensional space.

We find the $u$ and $v$ axes of the congruence, that are transversals of the straight lines $a, b, c, d$ using the method described in Section 2.If these transversals are real different (HLC) or real 
coincident (PLC), then the solution known from descriptive geometry is reduced to the constructing of a straight line passing through a given point $T$ and intersecting the found axes [1]. If the transversals $u$ and $v$ are imaginary, then they cannot be represented explicitly. In this case, the problem is reduced to the constructing a real straight line passing through $T$ and intersects the imaginary lines $u, v$. The following algorithm is proposed to construct such a straight line:

Step 1. We introduce into consideration auxiliary linear quadrics $\varphi_{1}(a, b, c)$ and $\varphi_{2}(a, b, d)$, which are completely defined by triples of generators $(a, b, c)$ and $(a, b, d)$, respectively. The imaginary straight lines $u, v$ (the ELC axes), being transversals of the given straight lines $a, b$, $c, d$, intersect with both triples of generators, therefore, the imaginary straight lines $u, v$ belong to both quadrics.

Step 2. Through the point $T$ indicated in the problem statement, draw an arbitrary plane $\Sigma$ and draw conical sections $f_{1}=\varphi_{1} \cap \Sigma, f_{2}=\varphi_{2} \cap \Sigma$ (Fig. 2, a). The construction of continuously drawn conics $f_{1}, f_{2}$ is performed using the special software [8]. The conics $\mathrm{f}_{1}, f_{2}$ passing through the groups of points $A, B, C$ and $A, B, D$, intersect each other at real points $A, B$ and at imaginary conjugate points $U \sim V$ (here $\mathrm{A}, \mathrm{B}, \mathrm{C}, \mathrm{D}$ are the intersection points lines $a, b, c, d$ with the plane $\Sigma$ ).

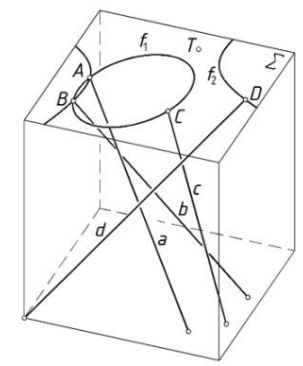

a)

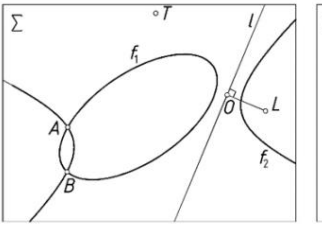

b)

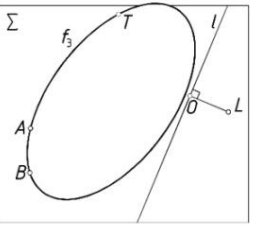

c)

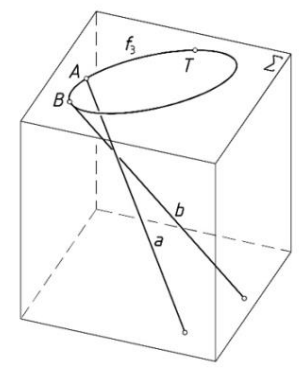

d)

Fig. 2 .Allocation of a straight line incident to the point $T$ from the ELK: a- initial data; bconstruction of marker $\{l, O L\}$ of imaginary points $U \sim V$; c- construction of a conic passing through imaginary points $U \sim V ; \mathrm{r}$ is determinant of a quadric containing the desired straight line.

Step 3. The imaginary straight lines $u, v$ belong to the quadrics $\varphi_{1}, \varphi_{2}$; therefore, the imaginary points of intersection of these straight lines with the plane $\Sigma$ coincide with the imaginary points $U \sim V$ of the intersection of the conic sections $f_{1}, f_{2}$.

Intersecting at real points $A, B$ and at imaginary conjugate points $U \sim V$, the conics $f_{1}, f_{2}$ establish on the line $l=U V$ the same elliptic involution $\sigma$ with imaginary double points $U, V$.The imaginary points $U \sim V$ are depicted by the marker $l\{O, L\}$, consisting of the center $O$ of the involution $\sigma$ and the Laguerre point $L$, from which the involution $\sigma$ is projected by the orthogonal pencil of lines (Fig. 2, b). The real straight line $l$ passing through the imaginary conjugate points $U \sim V$ is the homology axis connecting the conics $f_{1}, f_{2}$.

The construction of marker $l\{O, L\}$, conventionally representing the imaginary points $U \sim V$, is carried out in a known way [11,12]. The graphic representation of imaginary points $U \sim V$ in the form of a marker $l\{O, L\}$ allows using these points along with real points in subsequent constructive constructions.

Step 4. Let's introduce into consideration an auxiliary linear quadric $\varphi_{3}(a, b, t)$, where $t$ is the required straight line. The generators $a, b, t$ of this quadric, according to the condition of the problem , intersect with the imaginary axes $u, v$ of the elliptic congruence $\operatorname{Kr}(a, b, c, d)$, therefore, the axes $u, v$ are included in the composition of in the quadric $\varphi_{3}$.In the section of this quadric by the plane $\Sigma$, we obtain the conic $f_{3}$ passing through the real points $A, B, T$ and 
through the imaginary conjugate points $U \sim V$, given in the drawing by the marker $l\{O, L\}$ (Fig. 2,b). A projective computer algorithm [11], compiled according to [13, p. 147], is used to draw the conic f3,

Step 5. The continuously drawn conic $f_{3}$ and the rectilinear generators $a, b$ intersecting it at points $A, B$ uniquely determine the quadric $\varphi_{3}(a, b, t)$, which allows finding the desired straight line $t$ from the conditions of its incidence to the point $T$ and belonging to the family $a$, $b, \ldots$-forming quadrics $\varphi_{3}$ (Fig. 2, d). The problem has been solved.

\section{Extracting a surface from ELK}

Theorem 1. If an algebraic linear surface $\Theta$ of the order $k+2$ is given by a guiding curve $e^{k}$ of the order $\mathrm{k}$ and two intersecting rectilinear guides $u, v$ (real or imaginary conjugates) intersecting the plane of the curve $e^{k}$ at points $U, V$ (real or imaginary conjugates), then the line $U V$ (always real) is the axis of the pencil of planes intersecting the surface $\Theta$ along algebraic curves of the order $k$.

Proof. The line $U V$ intersects the guiding curve $e^{k}$ at two points (real or imaginary conjugate), and also intersects the rectilinear guides $u, v$, therefore, $U V$ is two coincident generators of the surface $\Theta$. The linear surface $\Theta$ is an algebraic surface of the order $k+2$, in the section of which by an arbitrary plane passing through $U V$, we obtain a curve that splits into the double line $U V$ and a curve of the order $k$. The theorem is proved.

The visualization of the ruled surface $\Theta$, extracted from the $\operatorname{ELC} \operatorname{Kr}(a, b, c, d)$ by immersing a guiding curve into it, is visualized on the basis of Theorem 1 and multiple repetition of Algorithm $1 \ldots 5$ described in Section 3.

Example. Let it be required to form a fourth-order linear algebraic surface $\Theta$, "stretched" on a space frame, given by a circle $r$ lying in the plane $\Sigma$, and four intersecting straight lines $a, b$, $c, d$ (Fig. 3, a). The condition of the problem is satisfied by the surface separated from the linear congruence $\operatorname{Kr}(a, b, c, d)$ by immersing the guiding circle $r$ into it. In accordance with Algorithm $1 \ldots 5$, the line quadrics $\varphi_{1}(a, b, c)$ and $\varphi_{2}(a, b, d)$ are introduced into consideration. Having drawn the auxiliary conical sections $f_{1}=\varphi_{1} \cap \Sigma, f_{2}=\varphi_{2} \cap \Sigma$, let's note that they intersect only at two real points A,B , therefore, the $u \sim v$ axes of the congruence $\operatorname{Kr}(a, b, c, d)$ are imaginary (the conics $f_{1}, f_{2}$ are not shown in Fig. 3 conventionally).

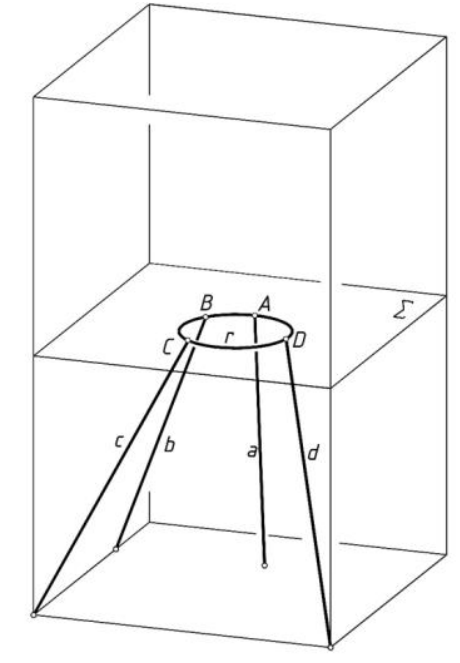

a)

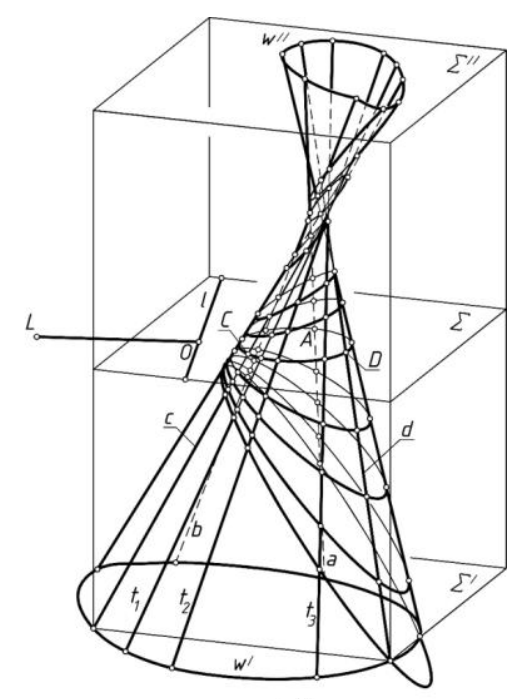

b)

Fig. 3. Selection of the surface from an ELC: $\mathbf{a}$ - initial data; b- surface frame and marker $\{l, O L\}$ of imaginary points of intersection of the ELC axes with the plane $\Sigma$ 
Find the marker $l\{O, L\}$ of the imaginary points $U \sim V$ of the intersection of the conics $f_{1}$ and $f_{2}$. At these points, the imaginary axes $u, v$ of the congruence $\operatorname{Kr}(a, b, c, d)$ intersect the plane $\Sigma$ (Fig. 3b). In accordance with Algorithm $1 \ldots 5$, mark several points on the guide circle $r$ and extract from $\operatorname{Kr}(a, b, c, d)$ several additional rectilinear generators $t_{i}(i=1,2,3, \ldots)$ of the constructed surface $\Theta$ passing through points marked on $r$.

According to Theorem 1, the planes passing through the straight line $l=U V$ intersect $\Theta$ in conical sections. A surface frame is obtained consisting of straight lines and curves of the second order (see Fig. 3, b).The planes $\Sigma^{\prime}, \Sigma^{\prime \prime}$ that do not pass through $l$ intersect $\Theta$ along curves of the fourth order $w^{\prime}, w^{\prime \prime}$. A visualization of the surface is shown in Fig.4.

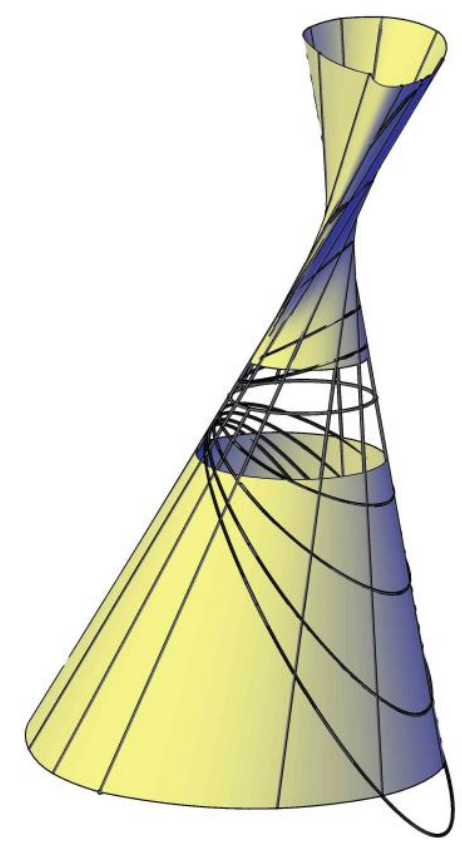

Fig. 4. Algebraic linear surface with imaginary axes (visualization)

\section{Specifying the congruence $K r(a, b, c, d)$ using a colline- ation}

The congruence $\operatorname{Kr}(a, b, c, d)$, given by the intersecting straight lines $a, b, c, d$, can be identically defined by the congruence $\mathrm{Kr}(\Pi \leftrightarrow \Pi$ ') of straight lines connecting the corresponding points of the collinear fields $\Pi \leftrightarrow \Pi^{\prime}$ ( provided that in collineation $\Pi \leftrightarrow \Pi^{\prime}$ the line $l=\Pi \cap \Pi^{\prime}$ corresponds to itself) [3, 14]. The $u, v$ axes of the congruence $\operatorname{Kr}(\Pi \leftrightarrow \Pi$ ') (real or imaginary) intersect the straight line $l$ at the real or imaginary conjugate points $U, V$. Consider two versions of the transition from the congruence $\operatorname{Kr}(a, b, c, d)$ to the identical congruence $\operatorname{Kr}\left(\Pi \leftrightarrow \Pi^{\prime}\right)$

\subsection{Variant 1 (one of these straight line is self-consistent)}

A linear congruence $\mathrm{Kr}(a, b, c, d)$ is given. Through one of the straight lines (for example, through $d$ ) we draw arbitrary planes $\Pi$, $\Pi$ '. The straight lines $a, b, c$ are cut out on planes $\Pi, \Pi^{\prime}$ points $A, B, C$ and $A^{\prime}, B$ ', $C^{\prime}$. We define a collinear correspondence of the fields $\Pi \leftrightarrow \Pi^{\prime}$ by three pairs of points $A \sim A^{\prime}, B \sim B$ ', $C \sim C^{\prime}$ and a straight line $d=\Pi \cap \Pi$ ', which we will assume to be self-consistent. In this case, the family of straight lines connecting the corresponding points of the fields $\Pi, \Pi^{\prime}$ is the linear congruence $\operatorname{Kr}(\Pi \leftrightarrow \Pi ')$ [3]. 
Let us show that the congruences $\operatorname{Kr}(a, b, c, d)$ and $\operatorname{Kr}\left(\Pi \leftrightarrow \Pi^{\prime}\right)$ coincide. The collineation $\Pi \leftrightarrow \Pi$ ' induces on the straight line d a projectivity with double points $U=\mathrm{U}^{\prime}, \mathrm{V}=\mathrm{V}^{\prime}$ (real or imaginary). The axes $u, v$ (real or imaginary) of the congruence $\operatorname{Kr}\left(\Pi \leftrightarrow \Pi^{\prime}\right)$ pass through these points and intersect with the straight lines $a, b, c$, therefore, the straight lines $u, v$ are transversals of the straight lines $a, b, c, d$, that is the axes of the congruence $\operatorname{Kr}(a, b, c, d)$. The coincidence of the congruences follows from the coincidence of the axes.

The proposed version of the transition from $\operatorname{Kr}(a, b, c, d)$ to $\operatorname{Kr}\left(\Pi \leftrightarrow \Pi^{\prime}\right)$ can be used to extract from the congruence $\operatorname{Kr}(a, b, c, d)$ the straight line $t$ passing through a given point $T$. Let's draw the plane $\Pi$ through the straight line $d$ and point $T$. Draw another (arbitrary) plane $\Pi$ ' through the straight line d. We mark the points of intersection $A \sim A^{\prime}, B \sim B^{\prime}, C \sim C^{\prime}$ of straight lines $a, b$, $c$ with planes $\Pi$, $\Pi^{\prime}$. In the collineation $\Pi(A, B, C) \leftrightarrow \Pi^{\prime}\left(A^{\prime}, B{ }^{\prime}, C^{\prime}\right)$ with the selfcorresponding line $\mathrm{d}$, we find the point $T^{\prime}$ corresponding to the point $T$. Through the points $T \sim T^{\prime}$ we draw the sought straight line $t$. The sought straight line is extracted from the congruence.

\subsection{Variant 2 (general case)}

A linear congruence $\operatorname{Kr}(a, b, c, \mathrm{~d})$ is given. Let us introduce into consideration the line quadrics $\varphi_{1}(a, b, c)$ and $\varphi_{2}(a, b, d)$ and an arbitrary plane $\Pi$ intersecting them. We draw conical sections $f_{1}=\varphi_{1} \cap \Pi, f_{2}=\varphi_{2} \cap \Pi$,passing through the triples of points $(A, B, C)$ and $(A, B, D)$. Here $A, B, C, D$ are the points of intersection of the straight lines $a, b, c, d$ with the plane $\Pi$.

The conics $f_{1}, f_{2}$ intersect at the points $\mathbf{A}, \mathbf{B}$ and at two more points $U, V$ (real or imaginary) through which the axes $u$ and $v$ (real or imaginary) of the congruence $\operatorname{Kr}(a, b, c, d)$ pass. We draw an arbitrary plane $\Pi$ ' through the straight line $l=U V$ (always real) and mark the points $A^{\prime}, B^{\prime}, C^{\prime}, D^{\prime}$ of intersection of rays $a, b, c, d$ with the plane $\Pi^{\prime}$. Let's define the collinear correspondence of the fields $\Pi \leftrightarrow \Pi^{\prime}$ by three pairs of the corresponding points $A \sim A^{\prime}, B \sim B^{\prime}, C \sim C^{\prime}$ and the self-corresponding straight line $l=\Pi \cap \Pi^{\prime}$. The collineation $\Pi \leftrightarrow \Pi^{\prime}$ induces on $l$ a projectivity with the double (self-corresponding) points $U, V$. The family of lines $a, b, c, \ldots$ connecting the corresponding collineation points $\Pi \leftrightarrow \Pi^{\prime}$ is the linear congruence $\mathrm{Kr}\left(\Pi \leftrightarrow \Pi^{\prime}\right)$, and the semi-quadric $\varphi_{1}(a, b, c)$ containing three straight lines $a, b, c$ of the congruence $\left.\operatorname{Kr}\left(\Pi \leftrightarrow \Pi^{\prime}\right)\right)$, all consists of the straight lines of this congruence.

Let us show that the straight line $d$ is included in the congruence $\operatorname{Kr}\left(\Pi \leftrightarrow \Pi^{\prime}\right)$.Let's suppose that the generators of the semi-quadrics $\varphi_{1}(a, b, c)$ and $\varphi_{2}(a, b, d)$ generate two different collineations. The double points $U, V$ coincide in these collineations. In addition, two common straight lines $a, b$ of the quadrics $\varphi_{1}, \varphi_{2}$ cut the same pairs of the corresponding points $A \sim A^{\prime}$, $B \sim B^{\prime}$ in the fields $\Pi, \Pi^{\prime}$. Four pairs of corresponding elements of the two collineations coincide, which means that the collineations' coincide. It follows from this that all generators of the semi-quadrics $\varphi_{1}(a, b, c)$ and $\varphi_{2}(a, b, d)$, and the straight line $d$, are included in the same congruence $\operatorname{Kr}\left(\Pi \leftrightarrow \Pi^{\prime}\right)$. The coincidence of the four rays $a, b, c, d$ of the congruences $\operatorname{Kr}(a, b$, c, d) and $\operatorname{Kr}\left(\Pi \leftrightarrow \Pi^{\prime}\right)$ implies the coincidence of the congruences themselves.

The proposed variant of the transition from $\operatorname{Kr}(a, b, c, d)$ to $\operatorname{Kr}\left(\Pi \leftrightarrow \Pi^{\prime}\right)$ can be used to extract a straight line $t$ passing through a given point $T$ from the congruence $\operatorname{Kr}(a, b, c, d)$. Draw through $T$ an arbitrary plane $\Pi$. Let's mark the points $A, B, C, D$ of the intersection of straight lines $a, b, c, d$ with the plane $\Pi$. Draw the sections $f_{1}(A, B, C)=\varphi_{1}(a, b, c) \cap \Pi, f_{2}(A, B, D)=\varphi_{2}(a$, $b, d) \cap \Pi$. Through the straight line $l$ connecting the points $U, V$ (real or imaginary) intersections of the conics $f_{1}, f_{2}$, draw an arbitrary plane $\Pi^{\prime}$ and mark the points $A^{\prime}, B^{\prime}, C^{\prime}$, $D^{\prime}$ where the straight lines $a, b, c, d$ intersect with the plane $\Pi^{\prime}$. We obtain the collineation $\Pi(A B C D) \leftrightarrow \Pi^{\prime}\left(A^{\prime} B^{\prime} C^{\prime} D^{\prime}\right)$ with double points $U, V$ lying on the self-corresponding straight line $l=\Pi \cap \Pi^{\prime}$.We find the point $T^{\prime}$ corresponding to the point $T$ in this collineation. The sought straight line $t$ passes through the points $T, T^{\prime}$. 


\section{Linear surface passing through two given conics}

A linear congruence given by collinear fields allows solving the practically important problem of constructing a linear surface passing through a pair of predetermined conic sections [14].

Theorem 2. If arbitrary conical sections $r, r^{\prime}$ with points $A, A^{\prime}$ are drawn in flat fields $\Pi, \Pi^{\prime}$, then there are only two variants of collineations $\Pi \leftrightarrow \Pi^{\prime}$ with a self-corresponding weakly invariant line $l=\Pi \cap \Pi^{\prime}$, in which $r \leftrightarrow r^{\prime}, A \leftrightarrow A^{\prime}$.

Proof. Draw the tangents $t_{A}, t_{A}^{\prime}$ to the given conics at the points $A, A^{\prime}$ (Fig. 5). From points $1=t A \cap l, 1^{\prime}=t^{\prime} A \cap l$ draw the tangents $t_{B}, t_{B}^{\prime}$. Marking the points $2=A B \cap l, 2^{\prime}=A^{\prime} B^{\prime} \cap l$, draw from these points the tangents $t_{C}, t_{D}, t_{C}^{\prime}, t_{D}^{\prime}$ to the conics $r, r^{\prime}$. We get the quadrilaterals $\left(t_{A}, t_{B}, t_{C}\right.$, $\left.t_{D}\right)$ and $\left(t_{A}^{\prime}, t_{B}^{\prime}, t_{C}^{\prime}, t_{D}^{\prime}\right)$ with vertices $(1,2, P, Q, M, N)$ and $\left(1^{\prime}, 2\right.$ ', $\left.P^{\prime}, Q^{\prime}, M^{\prime}, N^{\prime}\right)$, described near the conics $r, r^{\prime}$. According to Brianchon's theorem, the lines $A B, C D$ and $A^{\prime} B^{\prime}, C^{\prime} D^{\prime}$ connecting the touching points, pass through the points $3=Q P \cap M N, 3^{\prime}=Q^{\prime} P^{\prime} \cap M^{\prime} N^{\prime}$ of the intersection of the diagonals of these quadrilaterals (see Fig. 5).The lines $C D, A B$ and $C^{\prime} D^{\prime}, A^{\prime} B^{\prime}$, according to the theory of poles and polar, are incident to the points 1,2 and 1', 2' , therefore, in the planes $\Pi$ and $\Pi^{\prime}$ the complete quadrangles MNPQ and $\mathrm{M}^{\prime} \mathrm{N}^{\prime}$ are obtained $P^{\prime} Q^{\prime}$, carrying the harmonic groups of points on their sides [15].

Thus, the correspondence of the flat fields $\Pi$, $\Pi$ ', given by those indicated in Fig. 5 fours of tangents $\left(t_{A}, t_{B}, t_{C}, t_{D}\right)$ and $\left(t_{A}^{\prime}, t_{B}^{\prime}, t_{C}^{\prime}, t^{\prime}\right)$ or the fours of points $(M, N, P, Q),\left(M^{\prime}, N^{\prime}, P^{\prime}, Q^{\prime}\right)$, is a collineation (since straightness, incidence, and harmonism are preserved) that meets the conditions of the theorem. In this collineation the conics $r, r^{\prime}$ are mutually corresponding, and the straight line $l=12$ transforms into the straight line coinciding with it $l^{\prime}=1^{\prime} 2^{\prime}$. The pairs of the corresponding points $1 \sim 1^{\prime}, 2 \sim 2^{\prime}, \ldots$ do not coincide, therefore $l=l^{\prime}$ is a weakly invariant straight line. Only two collineation variants are possible: $\left(t_{A}, t_{B}, t_{C}, t_{D}\right) \leftrightarrow\left(t_{A}^{\prime}, t_{B}^{\prime}, t_{C}^{\prime}, t_{D}^{\prime}\right)$ или $\left(t_{A}, t_{B}, t_{C}, t_{D}\right) \leftrightarrow\left(t_{A}^{\prime}, t_{B}^{\prime}, t_{D}^{\prime}, t_{C}^{\prime}\right)$. The theorem is proved.

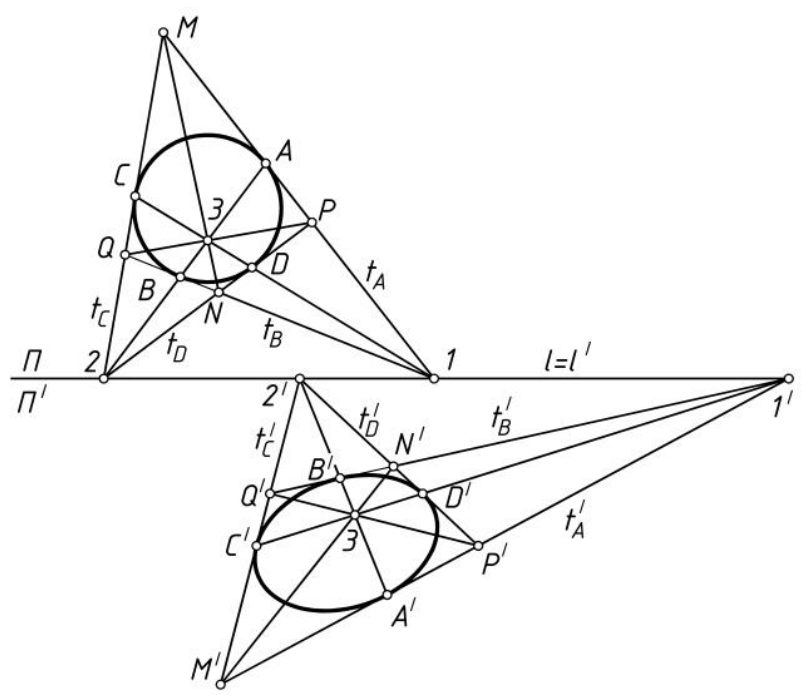

Fig. 5. For Proof of Theorem 2

\subsection{Algorithm for constructing rectilinear generators.}

On the basis of Theorem 2, the problem of constructing rectilinear generators of an algebraic surface of the fourth order passing through the conics $r, r^{\prime}$ and having a predetermined generator $A A^{\prime}$ is solved. Assuming that the collineation $\Pi \leftrightarrow \Pi^{\prime}$ is given by the drawn conics $r, r^{\prime}$ and a pair of corresponding points $A \leftrightarrow A^{\prime}$ on these conics, we obtain, according to Theorem 2, the congruence $\mathrm{Kr}\left(\Pi \leftrightarrow \Pi^{\prime}\right)$. To extract the desired surface from it, it is necessary to point to several points $\mathrm{Ti}$ on one of the cones and find the corresponding in the collineation $\Pi \leftrightarrow \Pi^{\prime}$ 
points $T_{i}{ }^{\prime}$, where $\mathrm{i}$ is the number of generators of the constructed surface required for accurate visualization.

Step 1. Draw the tangents $t_{A}, t_{A}^{\prime}$ to the conics $r, r^{\prime}$ at the points $A, A^{\prime}$. From the points $1=t_{A} \cap l$, $1^{\prime}=t_{A}^{\prime} \cap l$ draw the tangents $t_{B}, t_{B}^{\prime}$. From points $1=\mathrm{tA} \cap \mathrm{l}, 1^{\prime}=\mathrm{t}^{\prime} \mathrm{A} \cap \mathrm{l}$ draw the tangents $\mathrm{tB}, \mathrm{t} \mathrm{t}^{\prime} \mathrm{B}$. Marking the points $2=A B \cap l, 2^{\prime}=A^{\prime} B^{\prime} \cap l$, draw from these points the tangents $t_{C}, t_{C}^{\prime}$ (Fig. $6, \mathrm{~b}$ ). According to Theorem 2, the collinear correspondence of the fields $\Pi \leftrightarrow \Pi^{\prime}$ (with the selfcorresponding straight line $l$ and the mutually corresponding conics $r, r^{\prime}$ ) is completely determined by four straight lines $\left(t_{A}, t_{B}, t_{C}, A B\right) \leftrightarrow\left(t_{A}^{\prime}, t_{B}^{\prime}, t_{C}^{\prime}, A^{\prime} B^{\prime}\right)$, at which the points $C \leftrightarrow C^{\prime}$ are mutually correspondent.

Note for Step 1. Another possible collineation variant is generated by the corresponding straight lines $\left(t_{A}, t_{B}, t_{C}, A B\right) \leftrightarrow\left(t_{A}^{\prime}, t_{B}^{\prime}, t_{C}^{\prime \prime}, A^{\prime} B^{\prime}\right)$. In this case, the correspondence $C \leftrightarrow C^{\prime}$ is replaced by the correspondence $C \leftrightarrow C^{\prime \prime}$ (see Fig. 6, b).

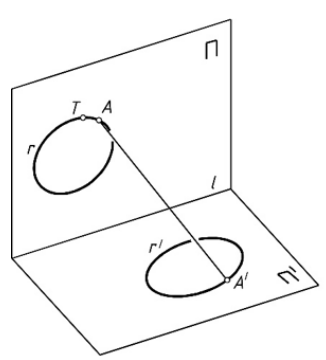

a)

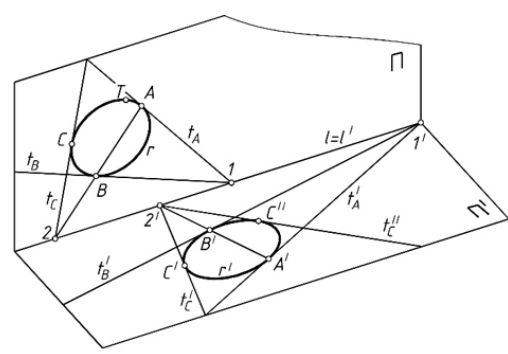

b)

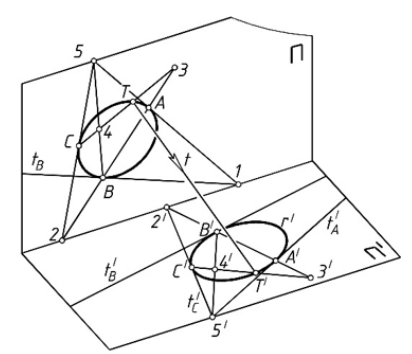

c)

Fig. 6. Constructing a surface passing through two conics: a- initial data; b- collineation $\Pi-$ $\Pi^{\prime}$ (two variants); c- extracting the generator $t$ from the congruence $\operatorname{Kr}\left(\Pi \leftrightarrow \Pi^{\prime}\right)$

Step 2. Through point $T$ an auxiliary straight line is drawn (for example, straight line $T C$ ) and point $3=T C \cap A B$ is marked on it (Fig. 6, c). From the condition preservation of a complex relation $(2 A B 3)=\left(2^{\prime} A^{\prime} B^{\prime} 3^{\prime}\right)$ we find point $3^{\prime}$.

Step 3. On lines $B 5$ and $B^{\prime} 5^{\prime}$ mark points 4 and $4^{\prime}$. From the condition of preserving the complex relation $(C T 43)=\left(C^{\prime} T^{\prime} 4^{\prime} 3^{\prime}\right)$ we find the point $T^{\prime}$. By direct verification on a computer model, we make sure that $T^{\prime}$ is incident to the conic $r^{\prime}$. Connecting the points $T$ and $T^{\prime \prime}$, we obtain the generator $t$ (see Fig. 6, c).

Multiple applications of the algorithm allow finding any number of rectilinear generators of the required surface. According to Theorem 1, the pencil of planes passing through $l$ intersects this surface along curves of the second order.

\subsection{Special case (planes $\Pi, \Pi^{\prime}$ are parallel)}

If planes $\Pi, \Pi$ ' are parallel, then the straight line $l$ becomes improper, and the projectivity degenerates into an affine correspondence of the point fields $\Pi \leftrightarrow \Pi^{\prime}$, since a simple relation and parallelism are preserved. For example, it is required to "pull" a linear surface onto a frame given by a rectilinear generatrix $A A^{\prime}$ and the conics $r, r^{\prime}$ lying in the parallel planes $\Pi(z x)$ and $\Pi^{\prime}\left(z^{\prime} x^{\prime}\right)$. By indicating in the fields $\Pi, \Pi^{\prime}$ three pairs of corresponding tangents $\left(t_{A}, t_{B}, t_{C}\right) \leftrightarrow$ $\left(t_{A}^{\prime}, t_{B}^{\prime}, t_{C}^{\prime}\right)$, we obtain the affinity $\Pi(\mathrm{r}, \mathrm{A}) \leftrightarrow \Pi^{\prime}\left(\mathrm{r}^{\prime}, \mathrm{A}^{\prime}\right)$ (Fig. $\left.7, \mathrm{a}\right)$. The straight lines connecting the corresponding points of the affine fields $\Pi, \Pi^{\prime}$ form the body of the linear congruence $\operatorname{Kr}\left(\Pi \leftrightarrow \Pi^{\prime}\right)$.

Extracting from $\operatorname{Kr}\left(\Pi \leftrightarrow \Pi^{\prime}\right)$ several generators of the required surface and using Theorem 1, we obtain the skeleton of an algebraic surface of the fourth order, consisting of a family of rectilinear generators and curves of the second order lying in parallel planes (Fig. 7, b). The surface visualization is shown in Fig. 8. 


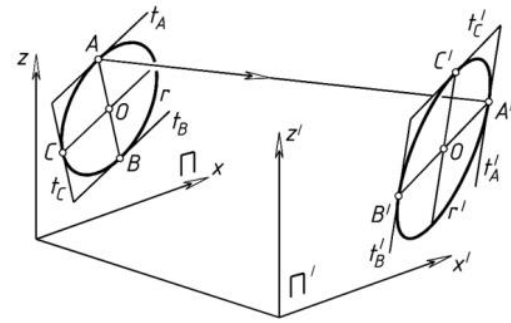

a)

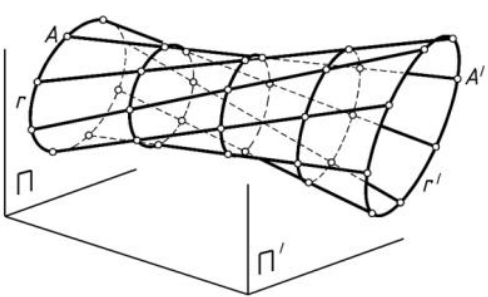

b)

Fig. 7. Special case (affine collineation $\left.\Pi \leftrightarrow \Pi^{\prime}\right)$ : $\mathbf{a}$ - initial data; $\mathbf{b}$ - surface frame is formed by conics lying in parallel planes

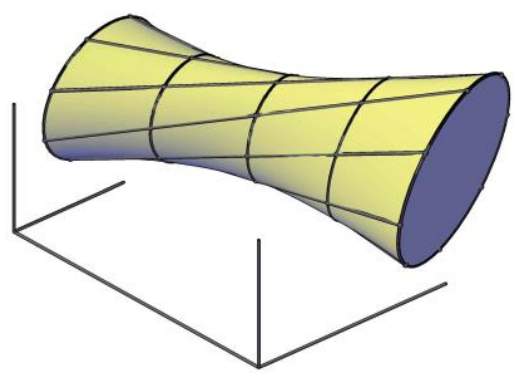

Fig. 8. Linear surface with imaginary axes (visualization)

Note to Section 6.2. If the conics $r, r^{\prime}$ are similar and similarly located, then the fourthorder algebraic surface degenerates into a linear quadric.

\subsection{Examples}

Example 1. Construct a fourth-order linear algebraic surface passing through the conics $r, r^{\prime}$ and having a predetermined generator $A A^{\prime}$ (Fig. 9).

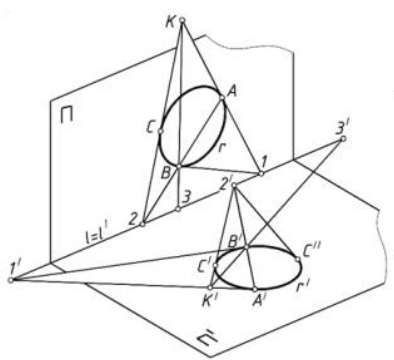

a)

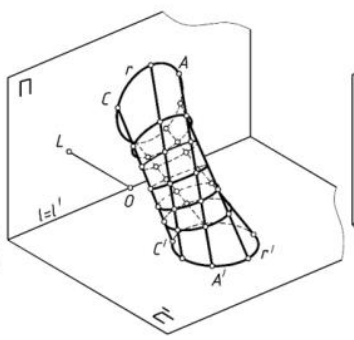

b)

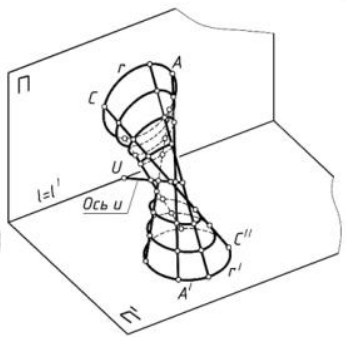

c)

Fig. 9. For Example 1: a- initial data; b- surface frame with imaginary axes (the first collineation variant $\left.\Pi \leftrightarrow \Pi^{\prime}\right)$; c- surface frame with real axes (the second collineation variant $\Pi \leftrightarrow \Pi \prime$ )

Draw the tangents to these conics and mark the points of tangency $A \sim A^{\prime}, B \sim B^{\prime}, C \sim C^{\prime}$ (or $\left.C \sim C^{\prime \prime}\right)$. According to Theorem 2, we obtain two variants of collineation: $\Pi(A B C K) \leftrightarrow$ $\Pi^{\prime}\left(A^{\prime} B^{\prime} C^{\prime} K^{\prime}\right)$ and $\Pi(A B C K) \leftrightarrow \Pi^{\prime}\left(A^{\prime} B^{\prime} C^{\prime \prime} K\right.$ ) (Fig. 9, a).

In accordance with the first option, triples of points $l(1,2,3) l$ ' $\left(1^{\prime}, 2\right.$ ', $\left.3^{\prime}\right)$ define projective point series on a common support $\mathrm{l}=\mathrm{l}$ 'with imaginary double points $\mathrm{U}=\mathrm{U}^{\prime}, \mathrm{V}=\mathrm{V}^{\prime}$ ', represented by the marker $l\{\mathrm{OL}\}$. The imaginary $u$ and $v$ axes of the congruence $\mathrm{Kr}\left(\Pi \leftrightarrow \Pi^{\prime}\right)$ pass through these points (Fig. 9, b). Using the algorithm $1 \ldots 3$ considered in Section 6.1, we find several rectilinear generators of the constructed surface. According to Theorem 1, the surface bears a family of conic sections, the planes of which pass through $l$ (see Fig. 9, b). 
Considering the second variant of collineation, we obtain a surface with real axes $u, v$ is obtained, which also carries a family of curves of the second order (Fig. 9, c). The visualization of both surfaces is shown in Fig. 10.

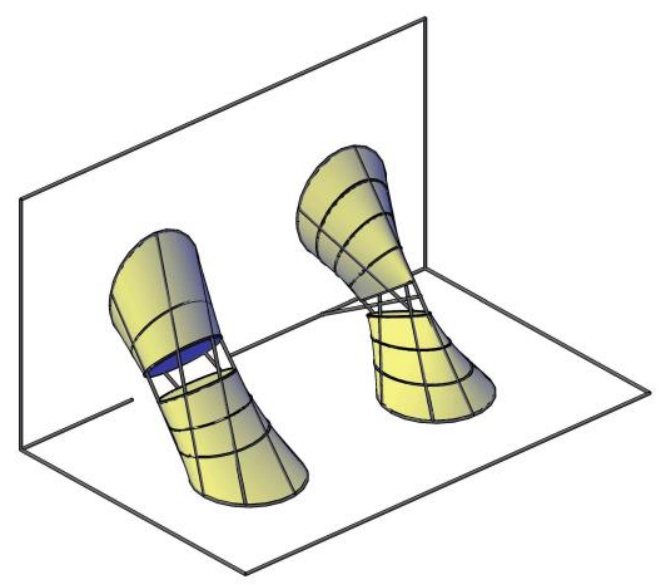

Fig. 10.Visualization (Example 1)

Example 2. Construct a fourth-order ruled algebraic surface passing through the hyperbolas $g, g^{\prime}$ and having a predetermined generator $A A^{\prime}$ (Fig. 11, a).We find several rectilinear generators of the constructed surface using Algorithm 1 ... 3 considered in Section 6.1. According to Theorem 1, the surface bears a family of conic sections (in this example, hyperbolas), the planes of which $\Pi_{1} \ldots \Pi_{4}$ pass through $l$ (Fig. 11, b). A visualization of the surface compartment is shown in Fig. 12.

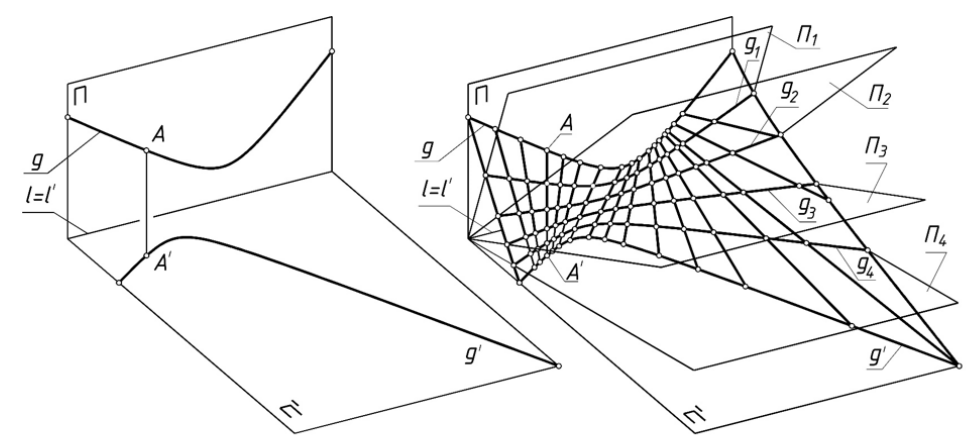

a)

b)

Fig. 11. For example 2: a- initial data; b- surface frame

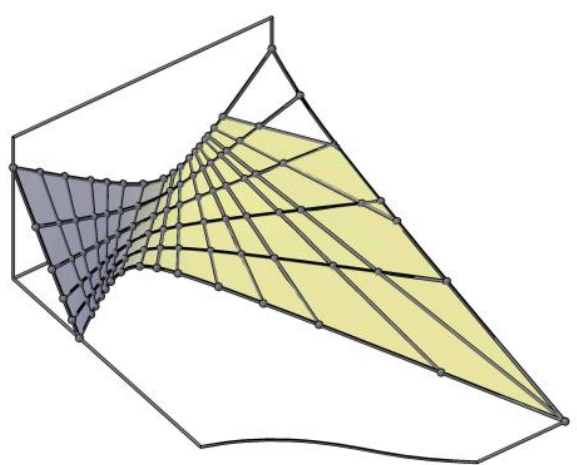

Fig. 12. Visualization (Example 2) 


\section{Biaxial linear surface with generators of constant length}

Let's consider a linear surface $\Omega$ formed by the motion of a straight line $a$, fixed points $M, N$ of which slide along intersecting straight lines $m, n$. We impose on the constructed surface the condition $|M N|=$ const (constancy of the length of the generators). Let us show that the surface $\Omega$ can be separated from the $\operatorname{GLK~} \operatorname{Kr}(m, n)$ by immersing a guiding ellipse into it, the eccentricity of which is determined only by the value of the angle between the axes $m, n$ of the congruence.

Theorem 3. If the ends of the segment $M N$ of a fixed length $\Delta$ slide along straight lines $m, n$ crossing at the angle $\alpha$, then the points of the segment describe ellipses lying in planes parallel to the straight lines $\mathrm{m}, \mathrm{n}$, and the midpoint of the segment $M N$ describes the middle (throat) ellipse $e$, the main axes $\delta_{1}, \delta_{2}$ of which are equal to $\delta_{1}=\operatorname{tg}(\alpha / 2) \sqrt{ }\left(\Delta_{2}-d_{2}\right)$, $\delta_{2}=\operatorname{ctg}(\alpha / 2) \sqrt{ }\left(\Delta_{2}-d_{2}\right)$, where $d$ is the distance between the axes $m, n$ (without proof).

Corollary 1. The ratio of the main axes $\delta_{1} / \delta_{2}=\tan ^{2}(\alpha / 2)$ of the throat ellipse is determined only by the value of the angle $\alpha$ between the axes $m, n$.

Corollary 2. The ellipse with the ratio of the principal axes $\delta_{1} / \delta_{2}=\operatorname{tg}^{2}(\alpha / 2)$, located symmetrically with respect to the directing lines $m, n$, separates an algebraic linear surface of order 4 with generators $M N$ of the constant length $|M N|=\Delta=\sqrt{ }\left(d_{2}+\delta_{1} \delta_{2}\right)$ from the hyperbolic linear congruence $\operatorname{Kr}(m, n)$.

In particular, if $\alpha=\pi / 2$, then, according to Corollary 1 , the throat ellipse degenerates into a circle. We get a double-symmetric linear surface with mutually perpendicular guides $\mathrm{m}, \mathrm{n}$, throat circle $\mathrm{r}$ and elliptical sections in planes parallel to the median plane (Fig. 13).

The technological advantage of such surfaces, which makes it possible to recommend them for use in architecture and construction, is that they are mounted from rectilinear beams or rods of the same standard size.

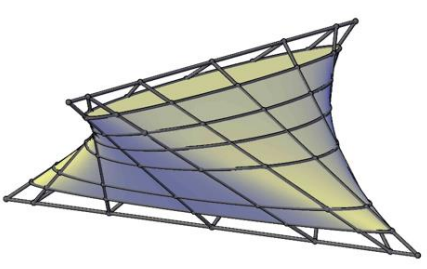

a)

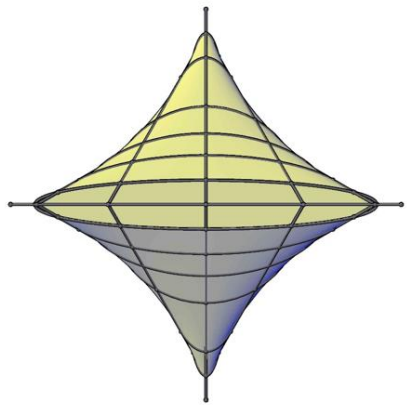

b)

Fig. 13. Visualization of a surface with mutually perpendicular axes and generators of constant length: a- axonometry; b- plan view

\section{Conclusion}

It is proposed a computer projective-graphic algorithm for the construction and visualization of an algebraic linear surface of the fourth order separated from the ELC by immersing a guiding conic into it. The surface is formed as a one-parameter set of straight lines intersecting two predetermined imaginary conjugate straight lines- ELC directrices (axes). The imaginary points of intersection of the imaginary axes of collineation with the real plane of the guiding conic are depicted with a special marker, which allows using imaginary points along with real ones when performing constructive constructions.

A theorem is proved on the existence of a pencil of planes intersecting a linear algebraic surface of order $k+2$ along algebraic curves of order $k$, which makes it possible to construct a skeleton of an algebraic surface of the fourth order from straight lines and curves of the second order. 
The variants of transition from a congruence given by four intersecting lines to a congruence of straight lines connecting the corresponding points of collinear fields are considered. Linear congruence given by collinear fields allows solving the practically important problem of constructing a fourth-order linear algebraic surface passing through a pair of predetermined conic sections.

A biaxial linear surface with constant-length generators is considered. It is shown that this surface is distinguished from a linear congruence with real axes by immersing a guiding ellipse into it, the eccentricity of which is uniquely determined by the angle between the congruence axes. The technological advantage of such surfaces, which makes it possible to recommend them for use in architecture and construction, is that they are mounted from rectilinear beams or rods of the same standard size. This makes it possible to recommend them for use in architecture and construction.

The computer projective-graphic algorithms proposed in the work make it possible to design architectural forms, floors, and shells that differ from traditional surfaces based on real axes, which expands the field of application of linear structures in architectural and construction design.

The figures presented in the article were made using a software product [8], which allows performing geometrically accurate construction of second-order curves (SOC) incidental to predetermined points and tangents. The algorithms outlined in [11] allow using of the program [8] to construct CWPs incident to both real and imaginary line elements.

\section{References}

1. Ivanov G.S. Teoreticheskie osnovy nachertatel'noj geometrii [Theoretical Foundations of Descriptive Geometry]. M: Mashinostroenie, 1998 [in Russian].

2. Suvorov F.M. Ob izobrazhenii voobrazhaemyh tochek i voobrazhaemyh pryamyh na ploskosti i o postroenii krivyh linij vtoroj stepeni, opredelyaemyh s pomoshch'yu voobrazhaemyh tochek i kasatel'nyh [On the image of imaginary points and imaginary lines on the plane and on the construction of curved lines of the second degree, determined using imaginary points and tangents]. - Kazan': Tipografiya imperatorskogo Universiteta, 1884 [in Russian].

3. Peklich V.A. Vysshaja nachertatel'naja geometrija [The Highest Descriptive Geometry]. M: ASV, 2000 [in Russian].

4. Peklich V.A. Mnimaya nachertatel'naya geometriya [Imaginary Descriptive Geometry]. M: ASV, 2007 [in Russian].

5. Hirsh A.G. Kompleksnaya geometriya - evklidova i psevdoevklidova [ Complex geometry - Euclidean and pseudo-Euclidean]. - M.: OOO «IPC "Maska"», 2013 [in Russian].

6. V. Korotkiy. Construction of a Nine-Point Quadric Surface // Journal for Geometry and Graphics, Austria, Volume 22 (2018), No. 2, pp. 183-193.

7. Korotkiy V.A. Komp'yuternaya vizualizaciya krivoj vtorogo poryadka, prohodyashchej cherez mnimye tochki i kasayushchejsya mnimyh pryamyh [Computer Visualization of a Curve of the Second Order passing through Imaginary Points and touching Imaginary Lines] // Scientific visualization, № 1, 2018, pp. 56-68 [in Russian].

8. Korotkij V.A. Programma dlya EVM "Postroenie krivoj vtorogo poryadka, prohodyashchej cherez dannye tochki i kasayushchejsya dannyh pryamyh" [ Construction of a curve of the second order passing through the data points and touching the data lines] // Svidetel'stvo o gosudarstvennoj registracii № 2011611961 ot 04.03.2011 $\mathrm{g}$ [in Russian]. 
9. Korotkiy V.A. Sinteticheskie algoritmy postroeniya krivoj vtorogo poryadka [Synthetic algorithms for constructing a second-order curve] // Vestnik komp'yuternyh i informacionnyh tekhnologij, № 11, 2014, pp. 20-24 [in Russian].

10. Korotkij V.A., Hmarova L.I. Universal'nyj komp'yuternyj konikograf [Universal computer conicograph] // Trudy 26-j Mezhdunarodnoj nauchnoj konferencii GraphiCon 2016, pp. 347-351 [in Russian].

11. Hirsh A.G., Korotkiy V.A. Graficheskie algoritmy rekonstrukcii krivoj vtorogo poryadka, zadannoj mnimymi elementami [Graphic Reconstruction Algorithms of the SecondOrder Curve, given by the Imaginary Elements] // Geometriya i grafika, № 4, 2016, pp. 19-30 [in Russian].

12. Hirsh A.G., Korotkij V.A. Mnimye tochki v dekartovoj sisteme koordinat [Imaginary points in a Cartesian coordinate system] // Geometriya i grafika, № 3, 2019, pp. 28-35 [in Russian].

13. Vol'berg O.A. Osnovnye idei proektivnoy geometrii [The Basic Ideas of Projective Geometry], Moscow-Leningrad: Gosudarstvennoe uchebno-pedagogicheskoe izdatel'stvo Publ., 1949 [in Russian].

14. Obuhova V.S. Konstruirovanie osevyh poverhnostej po napered zadannym usloviyam [Design axial surfaces according to predetermined conditions] // Prikladnaya geometriya i inzhenernaya grafika, № 6, 1968, pp. 23-34. Kiev: Budivel'nik [in Russian].

15. Chetveruhin N.F. Proektivnaya geometriya [Projective Geometry], M: Prosveshchenie, 1969 [in Russian]. 